\title{
Philosophiques
}

\section{Jean-Fabien Spitz, L'amour de l'égalité. Essai sur la critique de l'égalitarisme républicain en France 1770-1830, Paris, Vrin/EHESS, 2000, 286 p.}

\section{Christian Nadeau}

Volume 28, numéro 2, automne 2001

URI : https://id.erudit.org/iderudit/005674ar

DOI : https://doi.org/10.7202/005674ar

Aller au sommaire du numéro

Éditeur(s)

Société de philosophie du Québec

\section{ISSN}

0316-2923 (imprimé)

1492-1391 (numérique)

Découvrir la revue

Citer ce compte rendu

Nadeau, C. (2001). Compte rendu de [Jean-Fabien Spitz, L'amour de l'égalité Essai sur la critique de l'égalitarisme républicain en France 1770-1830, Paris, Vrin/EHESS, 2000, 286 p.] Philosophiques, 28(2), 453-457.

https://doi.org/10.7202/005674ar d'utilisation que vous pouvez consulter en ligne.

https://apropos.erudit.org/fr/usagers/politique-dutilisation/ 
Jean-Fabien Spitz, L'amour de l'égalité. Essai sur la critique de l'égalitarisme républicain en France 1770-1830, Paris, Vrin/EHESS, 2000, $286 \mathrm{p}$.

Avant tout autre commentaire, on ne peut que recommander la lecture de cet ouvrage, tant la lecture en est passionnante et la problématique on ne peut plus pertinente encore aujourd'hui. Sans espérer une comparaison entre le XVIII siècle et le nôtre, nombre des arguments exprimés dans cet ouvrage sont très proches de ceux qui sont formulés dans les débats contemporains au sujet de l'égalité. L'auteur défend l'opportunité de réfléchir aux différentes critiques qui furent faites aux doctrines de l'égalitarisme républicain, de la querelle des Anciens et des Modernes jusqu'aux thèses de l'humanisme marchand, telles qu'on les trouve chez un auteur méconnu, C.B. Dunoyer. J.-F. Spitz se garde donc de lire les seuls auteurs qui furent effrayés par une Révolution imminente ou par les effets de celle-ci. Il montre clairement que les adversaires de l'égalitarisme, pour bon nombre d'entre eux très conservateurs, n'en étaient pas pour autant des monarchistes convaincus. En somme, la critique de l'égalitarisme républicain n'est pas nécessairement antirépublicaine. Elle tente au contraire de montrer ce qui, dans l'égalitarisme, semble incompatible avec les acquis moraux et politiques de la Révolution. L'un des mérites du livre est de présenter les 
nuances d'une critique dont les prémisses empruntent autant à l'argumentaire conservateur qu'à des thèses affranchies du joug moral de l'Ancien Régime.

Jean-Fabien Spitz avait consacré la troisième partie de son dernier livre, $L a$ liberté politique (Paris, PUF, 1995) à la théorie de la liberté républicaine chez Rousseau. Cette étude était précédée de longs prolégomènes qui avaient pour tâche d'ancrer les recherches historiques de l'auteur dans une perspective plus contemporaine, soit les débats opposant la tradition libérale et les tenants d'une réactualisation du républicanisme. Avec P. Pettit pour la philosophie anglo-saxonne, J.-F. Spitz a été le premier à donner à cet effort en vue de construire une théorie républicaine de la liberté une cohérence et une unité qui jusqu'ici lui faisait défaut. C'est précisément cette recherche sur la spécificité de la théorie républicaine par rapport à la question de la liberté politique qui a conduit l'auteur à examiner plus en détail l'égalitarisme, faisant de celui-ci le compagnon indispensable de la liberté républicaine. Rappelons que pour Spitz, les citoyens d'une même société ne peuvent se considérer libres que s'ils sont assurés que les contraintes imposées à leur liberté sont nécessaires et d'égale valeur pour tous. Mais le plus important est que chaque individu soit assuré au même titre que les autres de pouvoir satisfaire ses désirs et ses aspirations. Le républicanisme ne nie pas le pluralisme en fonction de son principe d'égalité, il suppose au contraire que la pluralité des valeurs et donc la liberté politique sont conditionnels à l'égalité civique. Mais l'égalité civique ne saurait être sans une certaine égalité matérielle, puisque les inégalités de conditions doivent être considérées comme des entraves à la liberté au même titre que les injustices, non parce que ces dernières se résument à des différences de condition sociale, mais parce que ces différences rendent pratiquement caduques toutes mesures juridiques assurant à chacun son droit à la liberté.

Ces thèses, si claires dans le premier ouvrage de Spitz, sont malheureusement réduites à quelques vagues notes de bas de page dans son dernier opus. Certes, il n'était pas nécessaire de rappeler l'histoire et la théorie de l'égalitarisme républicain pour en commenter les diverses critiques à l'aube et au lendemain de la Révolution française. Le problème tient en fait à ce que nous ne savons plus très bien à qui s'adressent les critiques de l'égalitarisme étudiées par Spitz. Celui-ci nous résume très brièvement - trop - les thèses de Rousseau qu'il a examinées ailleurs. Mais il nous parle également des " disciples » de ce dernier, dont mis à part Mably peut-être, nous ne savons pratiquement rien. Encore une fois, il est facile de répondre que tel n'était pas le but de l'ouvrage. Spitz a moins cherché à reconstituer les éléments d'un débat que de réfléchir sur les arguments de la critique de l'égalitarisme. Mais alors pourquoi nous offrir cette suite de commentaires pointilleux sur des auteurs - le baron d'Holbach, N. A. Boulanger, Mme de Staël, Cabanis, Condorcet, Dunoyer sans que l'on sache très bien, non pas ce qu'ils ont en commun, mais au contraire ce qui les distingue ? Pourquoi était-il nécessaire de faire intervenir Mme de Staël ou Cabanis lorsque d'Holbach, Condorcet et Dunoyer auraient suffi à couvrir l'ensemble des thèses les plus importantes de l'anti-égalitarisme ? Il ressort alors que les subtilités et les distinctions entre les auteurs nous apparaîtraient plus clairement si nous disposions des thèses et des arguments précis contre lesquels fut élaborée la critique de l'égalitarisme. Ce problème, en apparence purement méthodologique voire littéraire, agace cependant et frustre le lecteur d'une étude qu'il aurait voulue plus approfondie. 
Une autre critique que l'on pourrait, me semble-t-il, adresser à ce livre est d'une autre nature, quoiqu'elle ne soit pas sans rapport avec ce qui précède. Une des hypothèses majeures du livre est que le programme politique dit " néo-classique » entendre républicain et égalitariste — n'a pas seulement servi de repoussoir au libéralisme moderne, mais que ce dernier doit en réalité beaucoup à son adversaire. Si le libéralisme se définit par l'aspiration à un régime juste et où chacun est libre d'agir comme bon lui semble sans être entravé dans son désir par une instance arbitraire, alors l'impartialité apparaît comme le principe clef des institutions politiques qui auront pour tâche d'assurer la réalisation effective de ce projet. Or, l'impartialité semble impossible sans l'égalité, et toute la question sera de savoir comment conjuguer l'une et l'autre sans annuler leur effet respectif. Là où le bât blesse, en raison peut-être de la méthode préconisée par Spitz, c'est qu'on voit mal quels étaient les effets pratiques visés ou obtenus par l'égalitarisme et/ou par ses adversaires. Nous assistons en grande partie ici à une querelle de principes dont les enjeux nous auraient pourtant laissé croire à autre chose que s'il s'agissait simplement de débats sur l'égalité de droit ou sur le patriotisme. Le nœud gordien de l'affaire est celui de l'égalité matérielle, et l'on se rend vite compte qu'une étude historique sur la question peut difficilement faire abstraction des débats entourant les mesures concrètes envisagées à ce sujet.

On aurait toutefois tort de voir dans cette étude l'affaire des seuls historiens. Comme le rappelle Spitz dans l'introduction de son livre, la question de l'égalité est, peut-être plus encore que celle de la justice, le véritable trait d'union des doctrines politiques contemporaines. On songe évidemment à R. Dworkin, mais également à A. Sen. Les positions de G.A. Cohen, si différentes soient-elles de celles de ces deux derniers, participent du même effort dans l'élaboration d'une conception acceptable de l'égalité politique ${ }^{1}$. À plusieurs égards, on retrouvera les accents des débats contemporains dans la littérature étudiée par Spitz. La question d'aujourd'hui demeure somme toute celle d'hier : si l'égalité est la condition d'une société juste, comment est-elle réalisable sans faire en sorte que les institutions nient les principes de liberté qui sont indissociables de ceux d'égalité ?

On sait qu'il revient à Tocqueville, plus encore qu'à tout autre, d'avoir montré le dilemme dans lequel s'enfermait l'homme moderne, assoiffé de liberté et d'égalité mais incapable de voir ce qui dans la dernière le conduisait à nier la valeur de la première. Comme Mme de Staël, Tocqueville insiste sur l'intolérance moderne - fruit indésirable mais inévitable de la Révolution - à l'égard de toute distinction sociale, ce qui conduit les hommes à appeler de leurs vœux le renforcement du pouvoir central de l'État. À l'hétéronomie de quelques-uns, les citoyens préfèrent la servitude de tous. Or, l'association de l'égalité des droits et celle des conditions est l'erreur même qui conduit au despotisme moderne où un Léviathan abstrait détruit toute tentative de distinction sociale et par là même tout effort d'autonomie personnelle. On connaît la réponse de Tocqueville, en partie reprise par ses contemporains, à ce dilemme : l'effort démocratique doit être protégé par un poison, celui de l'aristocratie, qui à

1. Cf. Kymlicka, Will, Contemporary political philosophy, 1992, tr. fr. Les théories de la justice, Paris/Montréal, Boréal/La Découverte, 1999, en particulier le chap. II consacré à l'« égalité libérale "; Sen, A., Inequality reexamined, Oxford, 1992 ; Dworkin, Richard, Sovereign virtue. The theory and practice of equality, Harvard, 2000 ; Cohen, G.A., "On the Currency of Egalitarian Justice », Ethics, 99/4, 1989, p.906-944. 
petite dose agira comme remède des maladies infantiles de l'égalitarisme démocratique. C'est un tel type de pensée que l'on retrouve aussi chez les détracteurs du libéralisme contemporain, lesquels voient dans l'égalitarisme une erreur, ce qui selon eux devrait nous conduire à préférer un principe de justice distributive fondée sur les mérites et ceci afin de s'assurer de conserver la liberté de chacun ${ }^{2}$.

En conclusion, il est difficile de voir en quoi exactement consistait, sinon dans les rêves de Rousseau et Mably, le programme républicain égalitariste qui fut la cible des auteurs étudiés par Spitz. Mais ce que nous démontre par contre ce livre, et il s'agit là d'un gain appréciable, c'est à quel point les auteurs aux origines des idées libérales avaient à cœur de répondre à l'égalitarisme républicain, ce dernier fût-il formulé de manière très vague et son usage pratique ne fût-il peut-être ressenti qu'au lendemain immédiat de la Révolution. Ce qui est très visible, c'est à quel point très rapidement, alors même que son programme n'était pas parfaitement défini, l'égalitarisme est perçu comme un grave danger, et comme le premier danger, dès lors que l'égalité matérielle est vue comme indissoluble de l'égalité juridique. C'est cette crainte que l'on ressent lorsqu'on voit à quel point le thème de l'égalité juridique est abordé avec une grande prudence, tant il est porteur de zizanie.

Dans son œuvre, J.W. Pocok, auquel Spitz doit beaucoup, avait posé la question de savoir si le projet de l'égalitarisme républicain — suivant lequel il faut concilier l'aspiration des hommes à la richesse matérielle et l'excellence morale, car sans cela la vertu républicaine serait toujours un idéal pour les Anciens, mais un cauchemar pour les Modernes - avait pu trouver une forme pratique satisfaisante. Par définition, il semble que ce projet ait été plus un instrument de critique politique qu'une véritable motivation en vue d'une refonte sincère des pratiques sociales et économiques. Ce projet n'en apparaît pas moins sous la plume de très nombreux auteurs. Il forme alors un courant de pensée que Pocock nomme l' " humanisme marchand ${ }^{3}$. Très tôt, pense Pocock, l'humanisme marchand a dû céder devant les arguments de Montesquieu suivant lequel le commerce fait gagner en sagesse et en pacification des échanges entre les hommes ce qu'il fait perdre en vertu. Tout échange économique implique de facto des rapports d'hétéronomie, lesquels adoucissent les humeurs mais corrompent les mœurs. Mais cette perte est le prix à payer pour la paix. Qui veut la paix ne prépare pas la guerre, mais s'incline et voit dans les armes de la politesse et de la civilité la puissance jadis recherchée au moyen du fer et au prix du sang. Or, dit Spitz, s'il est peut-être vrai que l'égalitarisme républicain dût vite céder le terrain devant ses critiques, il semble que l'aspiration égalitariste néo-classique ait été plus longtemps présente dans la pensée politique française, en attestent le nombre et la vigueur des critiques qui entendaient y répondre. À tel point, dit Spitz, que toute étude sérieuse de la pensée politique française des $\mathrm{XVIII}^{\mathrm{e}}$ et $\mathrm{XIX}^{\mathrm{e}}$ siècles doit avoir pour point de départ la question de l'égalité. Même si nous ne disposons pas de la totalité de l'argumentaire néo-classique, il suffit de relire Rousseau pour saisir combien aucun des arguments de l'égalitarisme républicain n'a été laissé pour compte par ses détracteurs. Tant et si bien que, si on accepte la démonstration de Spitz, le mouvement critique de l'égalitarisme semble avoir été mieux organisé que le républicanisme. À la rigueur, on pourrait presque dire que les critiques de

2. Spitz fait référence à Kekes, J., Against Liberalism, Cornell UP, 1997, chap.5.

3. Cf. en particulier Vertu, commerce et histoire, tr. fr. Paris, 1998. 
l'égalitarisme connaissaient mieux que quiconque les arguments de ce dernier, ce qui leur permettait d'anticiper les réponses des disciples de Rousseau et de mieux protéger leur propre système de pensée contre d'éventuelles attaques. Certes, le mouvement néo-classique ou républicain semble avoir vécu plus longtemps en France qu'en Angleterre ou en Amérique. Mais l'égalitarisme français est réduit chez Spitz à vivre au sein de sa critique et jamais indépendamment des thèses de ses détracteurs. Il en est comme le parasite, incapable de survivre autrement. Il n'est pas certain que ce soit là, loin s'en faut, la conclusion véritable de cet ouvrage. Tout y conduit pourtant, et c'est cette thèse qui, d'après moi, devrait maintenant faire l'objet d'une nouvelle étude.

CHRISTIAN NADEAU

Université de Montréal 